\title{
Lumen
}

Selected Proceedings from the Canadian Society for Eighteenth-Century Studies

\section{La tentation du théâtre dans le roman : analyse de quelques tableaux chez Sade et Richardson}

\section{Emmanuelle Sauvage}

Volume 20, 2001

URI : https://id.erudit.org/iderudit/1012309ar

DOI : https://doi.org/10.7202/1012309ar

Aller au sommaire du numéro

Éditeur(s)

Canadian Society for Eighteenth-Century Studies / Société canadienne d'étude du dix-huitième siècle

ISSN

1209-3696 (imprimé)

1927-8284 (numérique)

Découvrir la revue

Citer cet article

Sauvage, E. (2001). La tentation du théâtre dans le roman : analyse de quelques tableaux chez Sade et Richardson. Lumen, 20, 147-160.

https://doi.org/10.7202/1012309ar

Copyright (C Canadian Society for Eighteenth-Century Studies / Sociéte canadienne d'étude du dix-huitième siècle, 2001
Ce document est protégé par la loi sur le droit d'auteur. L'utilisation des services d'Érudit (y compris la reproduction) est assujettie à sa politique d'utilisation que vous pouvez consulter en ligne.

https://apropos.erudit.org/fr/usagers/politique-dutilisation/ 


\section{La tentation du théâtre dans le roman: analyse de quelques tableaux chez Sade et Richardson}

Roman et drame se voient assigner au XVIII ${ }^{\mathrm{e}}$ siècle des intentions morales identiques: émouvoir, éveiller la conscience des lecteurs et des spectateurs, les rendre meilleurs. Comment toucher la sensibilité d'un public qui a le goût des larmes? Comment susciter le pathétique? Rejetant respectivement l'héritage du roman baroque, héroïco-galant, et celui de la tragédie classique, trop éloignés du «vrai», de l'actualité sociale et morale du siècle, le genre romanesque et le théâtre doivent inventer de nouvelles formes esthétiques, ancrées dans de nouvelles réalités et répondant à de nouvelles attentes. On sait quel fut le rayonnement de Richardson en Europe dans la seconde moitié du siècle: longue est la liste des réécritures romanesques et dramatiques de Clarissa, nombreux sont ses admirateurs'. Or, ce qu'on loue dans ce roman, ce sont moins les qualités propres à la narration que le sens du drame et l'art de composer des tableaux ${ }^{2}$.

Qu'est-ce qu'un tableau romanesque? En France, les nouvelles conceptions dramaturgiques, l'invention du drame bourgeois par Diderot, le va-et-vient théorique entre la scène de théâtre, la peinture et l'écriture romanesque contribuent à redéfinir cette notion. Sade, à première vue si marginal dans le siècle, reprend à son compte l'héritage de cette triple dramaturgie. L'influence de l'Histoire de Miss Clarisse Harlove ${ }^{3}$ est manifeste dans Aline et Valcour, lorsque le marquis entreprend de dramatiser les scènes les plus intenses et les dialogues ${ }^{4}$. Ailleurs, il va plus loin dans l'hybridation formelle: le début des Cent Vingt Journées de Sodome ressemble beaucoup à une longue didascalie théâtrale ${ }^{5}$. L'analyse qui suit se limite à saisir la nature et le fonctionnement de quelques tableaux sadiens mis en parallèle, quand cela est possible, avec la version française de Clarissa ${ }^{6}$. Mais la notion de "tableau» nécessite au préalable quelques éclaircissements.

Le «tableau» a le pouvoir de suspendre momentanément l'action, d'arrêter le temps, de fixer les scènes les plus pathétiques d'un roman. C'est là un de ses premiers paradoxes, puisqu'il lui faut cristalliser ce qui ne se laisse pas docilement enfermer dans un cadre: les mouvements du 
cœur et du corps, le désordre des passions. Considéré comme un synonyme usuel du terme plus technique $\mathrm{d}^{\prime}$ «hypotypose ${ }^{7}$ ", il relève de la rhétorique et de la peinture, domaine auquel la doctrine de l'ut pictura poesis a tôt fait de le rattacher. À cette double appartenance s'ajoute le sens dramaturgique que lui donnent l'abbé Du Bos dans ses Réflexions critiques sur la poésie et sur la peinture ${ }^{8}$, puis Diderot dans ses Entretiens sur le Fils naturel ${ }^{9}$. Dénominateur commun à ces trois domaines artistiques - la peinture, le théâtre et l'écriture romanesque - le tableau demeure un concept ambigu: grâce à lui (ou à cause de lui), les drames se déguisent en romans et les romans en drames ${ }^{10}$. Beaumarchais a bien perçu les enjeux de cette contamination intergénérique quand il déclare dans son Essai sur le genre dramatique sérieux (1767) que «le Drame est la conclusion et l'instant le plus intéressant d'un roman quelconque ${ }^{11}{ }$. Cette définition servira de fil d'Ariane dans l'analyse des tableaux descriptifs que nous avons retenus.

La «mise en tableau» des personnages constitue l'une des manifestations de la théâtralité romanesque chez Sade et Richardson. Les descriptions $\mathrm{d}^{\prime}$ «instants» pathétiques, d'acmés dramatiques, de scènes figées dans l'intensité du moment abondent. Dans Clarissa, la pantomime est stéréotypée, les personnages sont décrits dans des postures théâtrales, avec une gestuelle et une mimique codifiées; les génuflexions, les implorations des mains et du regard, les expressions du visage, renvoient aux modes de représentation des passions et aux différentes attitudes de jeu répertoriées dans les traités d'art dramatique de l'époque ${ }^{12}$. Dans la célèbre scène où elle doit être jugée par Lovelace et ses complices pour avoir tenté d'acheter la confiance de Dorcas Wikes, Clarisse se conduit en héroïne tragique. Son redressement sublime en impose à ses accusateurs:

Toute l'assemblée demeure en silence à sa vue. Chacun est glacé d'étonnement ou de crainte. Moi-même je suis comme effrayé de sa situation \& de la mienne: le cœur me bat; l'embarras et la confusion me lient la langue, altèrent même mes forces. Elle est muette aussi quelques moments. Elle jette successivement un regard ferme, sur moi \& sur chaque personne de l'assemblée. Cette préparation achève de nous rendre immobiles. Ensuite, faisant quelques pas devant nous, dans la longueur de la chambre, \& retournant sur la même ligne, comme pour se donner le temps de chercher ses termes, ou de modérer son indignation, elle s'arrête en fixant les yeux sur moi: misérable Lovelace, commence-t-elle avec une force incroyable; ô le plus abandonné de tous les hommes! crois-tu que je ne pénètre pas ici ton infâme \& lâche complot ${ }^{13}$ ? $(\mathrm{HCH}, \mathrm{t}$. XI, lettre CCLIX, p. 258-259) 
À la fin de la scène, Clarisse menace de mettre fin à ses jours «en portant sur son cœur la pointe d'un canif ${ }^{14}{ }$ (p. 262), suscitant ainsi l'émoi de l'assemblée et, surtout, consacrant le triomphe de la vertu. Sa sortie est tout aussi théâtrale: "Après cette fière déclaration, elle a pris un des flambeaux qui étaient sur la table; \&, sans ajouter un seul mot, elle s'est retirée dans son appartement ${ }^{15} \gg$ (p. 267). Cette scène préfigure le «maudit songe» de Lovelace, qui imagine l'apothéose de Clarisse, suivie de sa propre chute $(\mathrm{HCH}, \mathrm{t}$. XIII, lettre CCCXXVII, p. 111-112). Elle marque la fin de son règne $d^{\prime}$ intrigant et la perte de ses pouvoirs de dramaturge.

Auparavant, tout au long de la première partie du roman - le «premier acte» $(H C H$, t. X, lettre CCXXIII, p. 132) -, le libertin aura mené l'intrigue de main de maître en usant avec succès de toutes les ficelles du théâtre. Dès le début, il crée de véritables rôles de comédie, impose à ses acteurs des répétitions, calcule le moindre détail scénique: de tenancière de maison close, madame Sinclair est promue au rang de veuve de lieutenant-colonel, les peu vertueuses Sally Martin et Polly Horton deviennent ses respectables nièces, Dorcas Wikes doit jouer la domestique éplorée, et il en va de même pour tous les personnages, recrutés, manipulés, transformés en acteurs par Lovelace. Lui-même est amené à composer différents rôles, à se déguiser, à faire preuve d'un grand contrôle de soi. Cet art consommé du jeu prouve à l'évidence l'hypocrisie fondamentale de ce personnage, héritier des libertins de Crébillon fils et précurseur de ceux de Laclos et de Sade, à la fois acteurs et metteurs en scène ${ }^{16}$.

Chez Sade, la maîtrise du corps passe par une mise en tableau comparable, qu'il s'agisse de donner un cadre aux effusions de larmes des victimes ou aux effusions du corps libertin. Dans Aline et Valcour, les larmes appellent les larmes; le spectacle d'Aline morte provoque la conversion de Dolbourg, le complice de M. de Blamont, tout comme Belford se dit "réformé» par la vision du martyre de Clarisse. Le tableau décrit en même temps l'exemplum et son effet pragmatique, souvent spectaculaire: quand Clarisse menace de se tuer, madame Sinclair et ses prétendues nièces avouent à Lovelace «que de leur vie elles n'avoient rien vu de comparable à cette scène, c'est-à-dire, apparemment, que jamais elles n'ont vu l'innocence si triomphante, \& le vice plus humilié ${ }^{17}$, $(H C H$, t. XI, lettre CCLIX, p. 264). Clarisse veut d'ailleurs que sa mort soit une leçon d'humilité à l'usage des vivants: «Apprenez, par mon exemple [...], comment tout finit ${ }^{18}{ }^{\prime}(\mathrm{HCH}, \mathrm{t}$. XIV, lettre CCCXLVII, p. 11-12).

Les deux romans s'achèvent sur une "conclusion" intensément dramatique, ultime «instant» pathétique de l'intrigue ${ }^{19}$. Ce qui demeu- 
rait chez Richardson une simple menace, l'objet d'un chantage, voire un cauchemar, advient dans Aline et Valcour:

Ô monsieur! quel spectacle!... Il m'est impossible de vous le peindre... cette chère maîtresse... cet ange du Ciel que je pleurerai toute ma vie... elle était à terre... elle était noyée dans son sang... elle avait devant elle les tresses des cheveux de madame, au milieu desquelles elle avait placé le portrait en miniature qu'elle possédait de cette mère respectable. Il est à croire qu'elle s'était poignardée devant ces chers objets de son cœur, et qu'à mesure que la perte de son sang lui avait ôté ses forces, elle était tombée sur ses genoux à la renverse; telle était la position où je la trouvai. L'arme qu'elle avait employée était une branche de longs ciseaux, dont elle se servait à sa toilette; elle avait séparé cette branche de l'autre, et se l'était enfoncée à trois reprises au-dessous du sein gauche; le sang avait abondamment coulé des trois blessures, et il ruisselait à grands flots dans la chambre; l'envie de la secourir, s'il en était temps, fut plus forte en moi que l'épouvante; je volai à elle mais elle était déjà froide [...]. Je la pris dans mes bras, en l'arrosant de larmes [...] (AV, p. 1092-1094).

Julie répond par ce tableau à l'injonction de Déterville: «Vous exigez des détails, quelque douloureux qu'ils soient, j'obéis» ( $A V$, p. 1079). De fait, la scrupuleuse domestique n'épargne guère le lecteur, invité à contempler avec une fascination mêlée d'effroi le spectacle de sa maîtresse suicidée. Ce tableau final est très développé en comparaison de ceux qui figurent ailleurs dans le roman. Il s'agit d'asséner le dernier coup en faveur de la vertu persécutée, afin d'en garantir la victoire. Seul le libertin M. de Blamont reste insensible au spectacle de sa fille morte: «Ce lugubre tableau ne lui inspira que de la colère... mais elle fut terrible...» $(A V$, p. 1094).

Julie, quant à elle, parvient à se ressaisir après le choc de la découverte macabre. La prétérition et la ponctuation expressive au début de la description traduisent son chagrin et son impuissance devant la mort de sa maîtresse. Le texte mime sa difficulté à exprimer l'horreur que lui inspire un tel spectacle. C'est ce moment chargé d'émotion que l'illustration du texte a voulu restituer. Le sous-titre de la dernière gravure reprend les premiers mots de Julie: «Oh Monsieur quel spectacle!» (p. 1093). On y voit la jeune domestique se détourner du funeste spectacle, le bras gauche replié sur les yeux, la bouche béante, le bras droit en l'air.

Dans le texte, le refus de voir, la prétendue incapacité de surmonter l'horreur du tableau est vite relayée par une description précise et circonstanciée, digne d'un procès-verbal. Aucun détail n'échappe au regard scrutateur de Julie. La description du corps immobile et exsangue, rendue par l'emploi du verbe «être» à l'imparfait («elle était à 
terre», «elle était noyée dans son sang»), fait place à une série de remarques et d'hypothèses concernant la position physique d'Aline, l'arme utilisée, la nature de la blessure, la quantité de sang déversée. On assiste à la reconstitution minutieuse de l'épisode du suicide, dépourvue de toute trace d'affliction de la part de la spectatrice. Les nombreux verbes d'action situent le texte dans une chronologie narrative très éloignée de l'immobilité décrite au début. Le tableau s'anime en scène. Une fois terminée la description des faits et gestes qui aboutissent au drame, Julie s'abandonne de nouveau à la douleur d'avoir perdu sa maîtresse. Le compte rendu froid et rationnel de cette mort solitaire est encadré par sa négation pathétique, toute de convention sous la plume de Sade. Le tableau ainsi brossé fait écho aux plus sombres pages du marquis.

La mise en tableau de la mort de Clarisse s'inscrit au contraire dans la durée pathétique; elle reproduit le déroulement des étapes qui conduisent lentement à l'issue fatale. La jeune femme est montrée en train de s'éteindre progressivement sous les yeux de ses proches. La description est effectuée du point de vue de Belford, toujours en fonction des attentes de Lovelace:

Vous aimez le détail: il faut vous peindre la scène qui s'est présentée à moi, lorsque je me suis approché du lit. M. Morden s'est attiré le premier mon attention. Il étoit à genoux, tenant une main de miss Harlove entre les siennes, le visage baissé dessus, \& la mouillant de ses larmes. De l'autre côté, madame Lovick, noyée dans les siennes, avoit la tête appuyée négligemment contre le chevet du lit; \& la tournant vers moi aussitôt qu'elle m'a vu: Ô M. Belford [...]. MadameSmith étoit debout près d'elle, les yeux levés, \& joignant aussi les mains, qu'elle pressoit l'une contre l'autre [...]. La garde étoit au-dessous de madame Lovick \& de madame Smith, la tête penchée [...]. Ses yeux paraissoient enflés à force de pleurer, quoiqu'elle dût être endurcie par l'habitude à ces tristes spectacles; \& les tournant vers moi, elle a paru m'inviter à joindre ma douleur à celle de l'assemblée. La servante de la maison, appuyée contre le mur, pressant des deux mains son tablier sur ses yeux, faisoit entendre encore plus distinctement ses sanglots $[\ldots]^{20}(H C H, t$. XIV, lettre CCCXLVII, p. 7-9).

Belford vient d'entrer dans la chambre et prend peu à peu connaissance des différentes attitudes des personnages présents. Ils sont regroupés autour du lit de Clarisse. Chacun est absorbé dans sa douleur, indifférent au nouveau venu, sauf Mme Lovick et la garde, qui tournent les yeux vers lui pour l'inviter en quelque sorte à entrer dans le tableau, à partager la douleur collective, bref à ne pas rester à l'écart comme un spectateur exclu de la scène ${ }^{21}$. D'abord silencieuse, la mourante se met à parler et lui avoue son chagrin de voir son entourage en pleurs («Le reste, je le vois bien, est plus fâcheux pour les spectateurs que pour $\operatorname{moi}^{22} »$, p. 10): 
par un glissement subtil, le pathos de la scène se déplace vers l'assistance. La jeune femme semble plus touchée par les témoignages de regret et d'affection à son égard que par sa propre mort, à laquelle elle s'est préparée et qu'elle attend comme une délivrance.

Sous l'œil attentif de ceux qui la veillent, elle s'interrompt et donne tous les signes de son départ. Puis elle se ranime, rouvre les yeux, semble reconnaître chacune des personnes à son chevet et prononce ses dernières paroles: «Enfin, levant les mains à demi, \& prononçant d'une voix confuse: Ciel! reçois une âme qui n'aspire qu'à toi, elle a rendu le dernier soupir ${ }^{23}$ » (p. 12). L'intensité de la scène, alors à son paroxysme, n'est pas très éloignée de la charge émotive présente dans ce que Pierre Frantz nomme au théâtre un «tableau-comble»:

il organise la plupart du temps l'ensemble des personnages autour d'une pantomime centrale, souvent silencieuse, qui manifeste un comble du pathétique ou un comble du sublime. Largement présent dans le drame et la comédie, il se rencontre pourtant aussi dans les tragédies ${ }^{24}$.

Dans la scène observée par Belford, le temps s'accélère insensiblement jusqu'à la mort de Clarisse. L'attente qui précède ce moment fatal renvoie néanmoins à la longueur d'un "plan-séquence» ou d'un «tableau-stase ${ }^{25}$ »: le drame, bien qu'imminent, n'a pas encore fait basculer le tableau dans le tragique. La représentation tabulaire est surtout adaptée à l'expression non verbale des sentiments. Pourtant, jusqu'à la toute fin, Clarisse ne cesse de parler, quitte à chercher ses mots et à balbutier.

Les manifestations du sensible peuvent aussi être orientées vers un cadre langagier plus spécifique de la théâtralité romanesque: la dramatisation des dialogues. Par sa présentation typographique, le discours direct, ou dramatisé, constitue un emprunt formel du roman au théâtre particulièrement remarquable. Richardson est l'un des premiers à utiliser le dialogue direct en indiquant les noms des personnages (ou leurs initiales) devant les répliques ou en les remplaçant par des tirets. $C^{\prime}$ est cette dernière méthode qui prévaudra dans la seconde moitié du siècle $^{26}$. Dans Clarissa, les dialogues sont modelés sur cette forme dramatique au moment où l'intrigue se resserre et que le piège se referme sur Clarisse. Quelques jours avant la nuit du viol, Lovelace multiplie les «machines» à dessein de faire tomber la vestale. Exaspéré par sa fuite, furieux de constater qu'elle se dérobe à son scénario, il est fermement décidé à se venger. La comédie qu'il fait jouer à ses acolytes lui en fournit les moyens.

L'abbé Prévost a conservé ce procédé de dramatisation des dialogues dans sa traduction, à cette différence près qu'il introduit le discours 
direct des personnages par une formule préliminaire en guise d'avertissement au lecteur. L'intérêt du procédé réside autant dans sa justification que dans son résultat. Il est intéressant de constater que les remaniements effectués dans la traduction française vont dans le sens d'un surenchérissement de la théâtralité du texte initial. Voici ce que l'abbé Prévost ajoute dans une lettre de Lovelace à Belford: «Il faut que je te [...] représente [le dialogue] en scène de comédie, tel que je l'ai entendu; c'est-à-dire, sous le nom de celle qui parle: sans quoi je serais embarrassé à te chercher des liaisons ${ }^{27}{ }$ ( $(H C H$, t. X, lettre CCXXVI, p. 208-209). Dans deux autres lettres, il fait dire ceci à Lovelace: «Ici, Belford, la nécessité m'oblige de revenir à la méthode du dialogue ${ }^{28} \gg(\mathrm{HCH}, \mathrm{t}$. XI, lettre CCXXXIV, p. 39), et aussi: «Mais il faut, Belford, que tu entendes les demandes \& les réponses, suivant la méthode que tu as goûtée dans quelques-unes de mes lettres ${ }^{29}{ }(\mathrm{HCH}, \mathrm{t} . \mathrm{XI}$, lettre CCXLI, p. 124). Pourquoi avoir introduit de la sorte ces scènes dialoguées?

Il est probable qu'à l'origine Richardson se dispense d'insérer des propositions incises - que Lovelace appelle des «liaisons» dans la version de Prévost - afin de garder intacte l'impression d'immédiateté que produit le discours direct: les raccords narratifs alourdiraient le style, ralentiraient le rythme, annuleraient toute illusion de vie ${ }^{30}$. Mais aussi réaliste et vivante qu'elle se veuille, cette technique ne va pas de soi dans la traduction de Prévost: en France, il est encore inhabituel de lire des dialogues romanesques sans jonction narrative. Étant donné que les conversations supposément retranscrites telles quelles contrastent beaucoup avec le reste du récit, on peut penser que le traducteur - et non Lovelace, bien sûr - a ressenti la «nécessité» de justifier le recours au discours direct, afin, vraisemblablement, de ne pas dérouter les lecteurs français, de la même manière qu'il censure le texte richardsonnien quand il le juge inconvenant ${ }^{31}$.

Dans Aline et Valcour, en l'espace d'une cinquantaine de pages réparties au début du texte, apparaissent une dizaine d'échanges rapportés suivant les conventions typographiques du texte théâtral écrit, avec, précédant chacune des répliques, tantôt le nom du locuteur, tantôt des tirets, tantôt un mélange des deux. Quelques notations de jeu suivent parfois directement le nom du personnage: «Écoutez, dit le garçon (en fermant la porte de crainte d'être entendu)» $(A V$, p. 816), «LE PRÉSIDENT, éclatant de rire» ( $A V$, p. 473), «LE PRÉSIDENT, ému» $(A V, \mathrm{p} .484)$, «M. DE BLAMONT, ému et balbutiant» $(A V$, p. 480$)$. Ces deux dernières indications correspondent aux rares fois où le libertin perd la face devant sa femme.

Dans une lettre à Valcour, Déterville restitue la discussion entendue par la Dubois entre Mirville et Delcour à propos de Sophie, en justifiant à son tour le recours à la «méthode» préconisée par Lovelace dans la 
traduction française: «comme leur dialogue est curieux, je vais te le transcrire mot à mot d'après les dépositions de la vieille, qui n'en a pas perdu une syllabe» $(A V, \mathrm{p}$. 461). Il continue un peu plus loin, après s'être permis une digression: «Poursuivons notre manière de rendre leur énergique conversation» ( $A V$, p. 462). Il semble bien que l'«énergique conversation» ait trouvé dans la dramatisation du discours un moyen tout aussi énergique de créer l'illusion de la présence des locuteurs. On assiste à un double effet de mise en scène du discours rapporté: les répliques sont non seulement insérées dans un espace textuel dénarrativisé, mimétique de l'écriture dramatique, mais elles sont aussi encadrées par le récit et par le format de la lettre. Le dialogue écrit devient tableau.

Le début des Cent Vingt Journées de Sodome offre une autre catégorie de tableau. L'«introduction» (CVJS, p. 70), ou l'«exposé» (CVJS, p. 40), selon les termes du narrateur sadien, semble calquée sur les discours de type didascalique, ce qui ne va pas sans ajouter de la confusion dans l'esprit du lecteur moderne. Il est d'autant plus difficile d'attribuer à ce texte une désignation générique précise qu'il entretient lui-même un certain flou sur la façon dont on pourrait le définir. Dès le début, le narrateur brouille les pistes en invoquant diverses appellations, tantôt équivalentes, tantôt différentes. Tour à tour, il est question d' «histoire» (CVJS, p. 15), de «roman» (CVJS, p. 24, 70), de «journal» (CVJS, p. 77, 311, $328,347)$, de «récit» au singulier (CVJS, p. 32, 39, 201) ou bien au pluriel (CVJS, p. 40), de «mémoires» (CVJS, p. 40), de «recueil» (CVJS, p. 181, $185,302)$, de «drame» (CVJS, p. 70), pour ne citer que quelques exemples. Les Cent Vingt Journées de Sodome participent effectivement de toutes ces catégories de texte et se rattachent en cela à maints genres littéraires ${ }^{32}$. Si la diversité interne de l'œuvre rend caduque toute tentative d'étiquetage, il importe toutefois de mettre l'accent sur le mouvement qui fait évoluer le récit-mémoires vers le drame. Les critiques ne s'y sont pas trompés, qui ont noté cette instabilité formelle:

Voici, en effet, un texte qui débute comme un roman historique, pour mettre en place une structure théatrale, se transformant en dialogue philosophique, qui s'amenuise en catalogue, pour se terminer en décompte des massacrés et des survivants. Voici un livre qui commence avec toute la pompe d'un roman historique pour aboutir au laconisme d'une simple soustraction ${ }^{33}$.

Nous en resterons à l'analyse de la «structure théâtrale» à laquelle Annie Le Brun fait référence.

L'«introduction» comporte, d'une part, une première partie descriptive, servant à présenter les arrangements préliminaires entre les libertins, les préparatifs de l'action, les portraits «étendus» des personnages, le «cabinet d'assemblée», et, d'autre part, une partie prescriptive, 
contenant les «Règlements» à respecter en ce qui concerne l'habillement et l'emploi du temps des personnages (CVJS, p. 62-63), sans oublier les obligations particulières des femmes, exhortées à l'obéissance par le duc de Blangis, ni l'avertissement du narrateur au lecteur. Cette nomenclature, qui se termine par une partie intitulée «Personnages du roman de l'École du libertinage», rappelle les instructions données dans les textes dramatiques sur le "costume de la pièce ${ }^{34}$ », ainsi que les indications plus ou moins longues que les auteurs ont coutume de fournir à l'usage du lecteur et des interprètes. Tout se passe comme si l'on avait affaire à une longue didascalie augmentée d'un prologue destiné à préparer le lecteur à la découverte des orgies programmées.

Toutefois, cette «introduction» n'est pas exempte d'éléments perturbateurs qui affaiblissent sa lisibilité. La présence envahissante des commentaires du narrateur et l'adjonction de microscènes greffées sur les développements descriptifs forment un agrégat verbal pour le moins composite. Ces digressions ont pour conséquence de diminuer l'efficacité des explications, de compromettre leur intelligibilité, et comportent le risque de fourvoyer le lecteur. D'ailleurs, à la fin, le narrateur éprouve le besoin de clarifier la présentation des personnages en la simplifiant:

Mais comme il y a beaucoup de personnages en action dans cette espèce de drame, que malgré l'attention qu'on a eue dans cette introduction de les peindre et de les désigner tous, on va placer une table qui contiendra le nom et l'âge de chaque acteur, avec une légère esquisse de son portrait, à mesure que l'on rencontrera un nom qui embarrassera dans les récits, on pourra recourir à cette table et, plus haut, aux portraits étendus, si cette légère esquisse ne suffit pas à rappeler ce qui aura été dit (CVIS, p. 70).

La «table» en question apparaît sous la forme d'une liste des personnages tout à fait comparable à celle des dramatis personæ qui précèdent généralement le texte des pièces de théâtre ${ }^{35}$. Le procédé n'est pas nouveau: Richardson aussi place au début de Clarissa une liste des noms des personnages principaux. Toutefois, elle se limite à signaler leurs liens familiaux et leurs rôles. Chez Sade, en plus de mentionner les noms des personnages en lettres majuscules, la liste indique leur âge, leurs liens de parenté, leur statut social et surtout leurs caractéristiques physiques et morales les plus remarquables. La disposition typographique et synoptique des informations, la concision du style, les ellipses verbales, ou bien l'emploi de l'indicatif présent, la relative neutralité du narrateur constituent des critères d'identification de cette liste au modèle didascalique $^{36}$. Voici, par exemple, la version raccourcie du portrait du duc de Blangis: «LE DUC DE BLANGIS, fait comme un satyre, doué d'un membre monstrueux et d'une force prodigieuse. On peut le regarder 
comme le réceptacle de tous les vices et de tous les crimes. Il a tué sa mère, sa sœur et trois de ses femmes» (CVJS, p. 70).

Les portraits «étendus» de la première série sont classés, comme ceux de la «table», en fonction des catégories auxquelles appartiennent les personnages: d'un côté figurent les libertins, leurs femmes-épouses, les historiennes et les servantes, de l'autre, le "sérail des jeunes filles», le «sérail des jeunes garçons» et les «huit fouteurs». Les deux séries diffèrent en longueur et en contenu: la seconde vient récapituler la première, les longs développements descriptifs du début font place à une galerie de portraits synthétiques qui tient en quelques pages à la fin de l'«introduction». Outre son aspect didactique, la «table» qui dresse la liste de ces portraits contribue à les dramatiser en leur donnant un nouveau cadre, débarrassé de toutes les scories narrativo-axiologiques qui venaient pour ainsi dire parasiter les portraits «étendus». La «table» se fait à son tour tableau.

Tableaux descriptifs, tableaux dialogiques, «table» didascalique: tous ces procédés, qu'ils soient explicitement désignés ou non par les auteurs, concourent à faire du roman un «mimotexte ${ }^{37}$ » théâtral. Le drame y est constamment mis en abyme, que ce soit par l'insertion de motifs emblématiques, tels que la représentation de l'Orpheline de Thomas Otway à laquelle Clarisse et Lovelace assistent $(\mathrm{HCH}$, t. VIII, p. 162, 169, 202), et celles du Père de famille de Diderot (CVJS, p. 721, 953, 966), ou, plus généralement, que ce soit par l'introduction de tableaux immobilisant les scènes les plus intéressantes. Le tableau romanesque, au-delà de son sens métaphorique, est l'instrument clé de la représentation du théâtre dans le roman; il est tableau de tableau. D'un point de vue strictement formel, le dialogue dramatisé et l'écriture didascalique sont plus proches du texte dramatique que les tableaux descriptifs. La théâtralité romanesque se joue entre ces deux pôles: d'un côté, le texte propose la mise en image verbale et iconique d'une théâtralité concentrée dans ou sur les «instants les plus intéressants» et, de l'autre, il reprend certaines des conventions d'écriture propres au genre dramatique, selon le principe de la «mimésis formelle ${ }^{38}$ ». Il semblerait que le roman doive imiter le théâtre pour gagner ses lettres de noblesse et être reconnu comme un genre à part entière.

Mais les rapports de subordination que l'on croirait unilatéralement établis du roman envers le théâtre ne sauraient cacher la réciprocité qui les unit en profondeur. Le roman tire son expressivité de la stylisation dramatique, en choisissant les moments les plus forts et en feignant de rapporter le discours des personnages tel qu'il a été entendu, quand le moment est «le plus intéressant»; le drame, lui, prétend «faire vrai» en représentant, comme un certain roman, les aléas de l'existence, considérée dans ses petits et grands événements. Il s'agit d'un double leurre, 
car, en fin de compte, les deux types de texte se nient chacun - et se nient mutuellement - comme œuvres de fiction.

\section{EMMANUELLE SAUVAGE}

\section{Notes}

1 À titre d'exemples, voir Diderot, Éloge de Richardson, dans CEuvres, édition établie par Laurent Versini, Paris, Robert Laffont, coll. «Bouquins», 1996, t. IV, p. 155-168; Laclos, pour qui «Clarisse [...] est celui des romans où il y a le plus de génie» (CEuvres complètes, texte établi, présenté et annoté par Laurent Versini, Paris, Gallimard, coll. «Bibliothèque de la Pléiade», 6, 1979, p. 469); Sade, Idée sur les romans, précédant les Crimes de l'amour, nouvelles hérö̈ques et tragiques, texte établi et présenté par Michel Delon, Paris, Gallimard, coll. «Folio», 1817, 1987, p. 38-40.

2 Richardson qualifie Clarissa de «dramatic narrative». Voir Godfrey F. Singer, The Epistolary Novel, New York, Russell and Russell, 1963, p. 62, cité par William J. Palmer, «Two Dramatists: Lovelace and Richardson in Clarissa», Studies in the Novel, 5, 1, printemps 1973, p. 20 n. 2. Diderot déclare pour sa part que «Paméla, Clarisse et Grandisson sont trois grands drames» (op. cit., p. 158).

3 Sade possédait dans sa bibliothèque de La Coste les romans du maître anglais traduits en français. C'est ce que montre l'inventaire dit «de 1778», dans Maurice Lever (édit.), Papiers de famille, Paris, Librairie Arthème Fayard, coll. «Bibliothèque Sade», 1995, t. II, p. 666-667. Clarissa est diffusée en France grâce à une première traduction par l'abbé Prévost, bien que le texte ait été coupé, remanié, dépossédé de son style original, selon beaucoup de critiques et d'historiens de la littérature: voir à ce sujet Colette Cazenobe, le Système du libertinage de Crébillon à Laclos, Studies on Voltaire and the Eighteenth Century, 282, 1991, p. 164-170. Toutefois, les libertés que prend le traducteur avec le texte de Richardson apportent des éléments intéressants pour l'analyse de la théâtralité du roman, aussi défiguré soit-il en français.

4 Voir aussi la Philosophie dans le boudoir (1795): cependant, nous n'avons pas retenu ce texte parce qu'il est entièrement dialogué.

5 Notre édition de référence est celle établie par Michel Delon (Sade, CEuvres, Paris, Gallimard, coll. «Bibliothèque de la Pléiade», 371, 1990, t. I). Les citations d'Aline et Valcour (1795) et des Cent Vingt Journées de Sodome (1785) seront suivies des sigles $A V$ et $C V J S$, ainsi que des numéros des pages. Le rapprochement entre ces deux romans et la production théâtrale sadienne serait sans doute éclairant, car il permettrait de s'interroger sur les ressources dramatiques mises à contribution de part et d'autre, mais une telle démarche excéderait le cadre que nous nous sommes fixé, à savoir l'étude du théâtre dans le roman.

6 Lettres angloises, ou Histoire de Miss Clarisse Harlove (1751), traduction de l'abbé Prévost, nouvelle édition augmentée de l'Éloge de Richardson, des Lettres posthumes et du Testament de Clarisse, Paris, Libraires associés, 1777, 14 tomes en 7 volumes. Les citations de ce texte seront suivies du sigle $\mathrm{HCH}$, puis des numéros du tome, de la lettre et des pages. Notre édition anglaise de référence est la suivante: The Clarissa Project. Samuel Richardson, Clarissa. Or, The History of a Young Lady, 
réimpression de la troisième édition (1751) en huit volumes, avec une introduction de Florian Stuber et une notice bibliographique de O. M. Brack, Jr., New York, AMS Press, 1990. Comme la numérotation des volumes et des lettres de l'édition française est différente de celle de l'édition anglaise, nous indiquerons en notes les numéros du volume, de la lettre et des pages qui correspondent au texte anglais.

7 "C'est lorsque, dans les descriptions, on peint les faits dont on parle comme si ce qu'on dit était actuellement devant les yeux; on montre, pour ainsi dire, ce qu'on ne fait que raconter; on donne en quelque sorte l'original pour la copie, les objets pour les tableaux» (Du Marsais, Traité des tropes, Paris, le Nouveau Commerce, 1977, p. 110).

8 «Une tragédie renferme une infinité de tableaux» (Réflexions critiques sur la poésie et sur la peinture, Paris, École nationale supérieure des beaux-arts, coll. «Beaux-arts histoire», 1993, p. 136).

9 «Une disposition [des] personnages sur la scène, si naturelle et si vraie que, rendue fidèlement par un peintre, elle me plairait sur la toile, est un tableau» (op. cit., p. 1136).

10 Diderot, tout en se défendant bien de confondre les genres, est l'auteur de drames remplis de péripéties, rebondissements et coups de théâtre censés imiter les fluctuations de la vie, tandis que la plupart de ses romans, eux, puisent à l'esthétique du tableau: «Qu'est-ce donc que le vernis romanesque qu'on reproche à quelques-unes de nos pièces? - Un ouvrage sera romanesque, si le merveilleux naît de la simultanéité des événements [...]; et surtout si l'enchaînement des événements y est trop extraordinaire et trop compliqué» (op. cit., p. 1297). À propos de la "romanisation» du drame et de la théâtralité romanesque, voir Henri Coulet, «Le roman théâtral», dans Claude Lachet (édit.), les Genres insérés dans le roman. Actes du colloque international du 10 au 12 décembre 1992, Lyon, Université Jean-Moulin, Lyon III, C.E.D.I.C., [1993], vol. IX, p. 187-200; Jean-Pierre Sarrazac, «Le drame selon les moralistes et les philosophes», dans Jacqueline de Jomaron (édit.), le Théâtre en France, Paris, Armand Colin, 1988, p. 333-338; Isabelle Vissière, «Une originalité de Diderot: le 'texte-image'», dans Diderot. Les beaux-arts et la musique. Actes du colloque international tenu à Aix-en-Provence les 14, 15 et 16 décembre 1984, Aix-en-Provence, Université de Provence, Centre aixois d'études et de recherches sur le XVIII ${ }^{\mathrm{e}}$ siècle, 1986, p. 109-122.

11 Beaumarchais, Théâtre complet, suivi des Lettres relatives à son théâtre, texte établi et annoté par M. Allem et P.-Courant, Paris, Gallimard, coll. «Bibliothèque de la Pléiade", 22, 1957, p. 8.

12 Voir Anne Patricia Williams, «Description and Tableau in the Eighteenth-Century British Sentimental Novel», Eighteenth-Century Fiction, 8, 4, juillet 1996, p. 471 et suiv.

13 The Clarissa Project, op. cit., vol. VI, lettre XIII, p. 61.

14 Ibid., p. 62.

15 Ibid., p. 66.

16 Voir à ce propos Laurent Versini, Laclos et la tradition, Paris, Klincksieck, 1968, p. 91-94 et 481-519; William J. Palmer, loc. cit., p. 7-13; Colette Cazenobe, op. cit., p. 244-256; Bernard Bray, «L'hypocrisie du libertin», dans Laclos et le libertinage, Paris, Presses universitaires de France, 1983, p. 97-109. 
17 The Clarissa Project, op. cit., vol. VI, lettre XIII, p. 64.

18 Ibid., vol. VIII, lettre I, p. 6.

19 La représentation tragique de la mort de la bien-aimée en fin de roman est un topos bien connu de la littérature française du XVIII ${ }^{\mathrm{e}}$ siècle: les Lettres persanes, Manon Lescaut, la Nouvelle Hélö̈se, l'Ingénu et Paul et Virginie en fournissent les exemples les plus célèbres.

20 The Clarissa Project, op. cit., vol. VIII, lettre I, p. 3-4.

21 En ce qui concerne le domaine de la peinture, Michael Fried a analysé la problématique de l'inclusion et de l'exclusion du spectateur dans la Place du spectateur: esthétique et origines de la peinture moderne, traduction de Claire Brunet, Paris, Gallimard, coll. «Essais / Gallimard», 1990, 264 p. Ill.

22 The Clarissa Project, op. cit., vol. VIII, lettre I, p. 5.

23 Ibid., p. 7.

24 Pierre Frantz, l'Esthétique du tableau dans le théâtre du XVIII siècle, Paris, Presses universitaires de France, coll. «Perspectives littéraires», 1998, p. 168.

25 Ibid., p 157-166.

26 Voir Vivienne Mylne, «Prévost et la traduction du dialogue dans Clarissa de Richardson», Cahiers Prévost d'Exiles, 3, 1986, p. 45.

27 Bien que ce rajout de Prévost ne corresponde pas au texte anglais, nous donnons quand même les références de la lettre à laquelle il se rapporte: The Clarissa Project, op. cit., vol. V, lettre X, p. 116. Il en va de même pour les deux citations qui suivent.

28 Ibid., vol. V, lettre XVIII, p. 171.

29 Ibid., vol. V, lettre XXVI, p. 241.

30 Dans sa préface à Clarissa, l'auteur exprime le désir de créer l'illusion de l'immédiateté dramatique grâce à ce qu'on peut appeler, selon lui, «instantaneous Descriptions and Reflections [...]; as also [...] affecting Conversations». Il continue ainsi: «Much more lively and affecting [...] must be the Style of those who write in the height of a present distress [...] than the dry, narrative, unanimated Style of a person relating difficulties and dangers surmounted, can be [...]» (op. cit., vol. I, «Preface», p. viii).

31 Vivienne Mylne envisage plutôt la question sous l'angle de la difficulté qu'aurait éprouvée le traducteur à «fournir des verbes de jonction tout au long de la conversation" (loc. cit., p. 47)

32 Michel Delon analyse ces modèles de référence dans CVIS, «Notice», p. 1124-1126.

33 Annie Le Brun, Soudain un bloc d'abîme, Sade, en tête des CEuvres complètes du marquis de Sade, édition mise en place par A. Le Brun et J.-J. Pauvert, Paris, Éditions J.-J. Pauvert, 1986, p. 42 (nous soulignons).

34 Pierre Frantz consacre un chapitre à cette question (op. cit., p. 87-114).

35 Voir Patrice Pavis, Dictionnaire du théâtre, Paris, Éditions sociales, 1980, p. 133-134.

36 Voir Jacqueline Viswanathan, «Spectacles de l'esprit», Poétique, 75, 1988, p. 376-377.

37 Gérard Genette désigne par «mimotexte tout texte imitatif, ou agencement de mimétismes» repérables dans un texte mis en relation avec un «modèle» perçu en 
amont (Palimpsestes. La littérature au second degré, Paris, Seuil, coll. «Points essais», 257, 1982, p. 106).

38 Michal Glowinski est à l'origine de ce concept: «La mimésis formelle ne se fonde [...] jamais sur une assimilation totale ou sur un transfert complet des principes structurels d'un mode d'expression dans un autre. Elle se ramène plutôt à un ensemble d'analyses qui doivent suggérer l'identité, mais en même temps témoigner de l'impossibilité d'atteindre cette identité. La mimésis formelle ne consiste donc pas, pour une forme littéraire, à se soumettre à des formes d'expression extra- ou paralittéraires» («Sur le roman à la première personne», dans Esthétique et poétique, textes réunis et présentés par Gérard Genette, Paris, Seuil, coll. «Points essais», 249, 1992, p. 235). Voir aussi Jacqueline Viswanathan, loc. cit., p. 374-375. 